\title{
Hadronic Molecules and Scattering Amplitudes from the Nonrelativistic Quark Model
}

\author{
T. Barnes \\ Physics Division and Center for Computationally Intensive Physics \\ Oak Ridge National Laboratory, Oak Ridge, TN 37831-6373
}

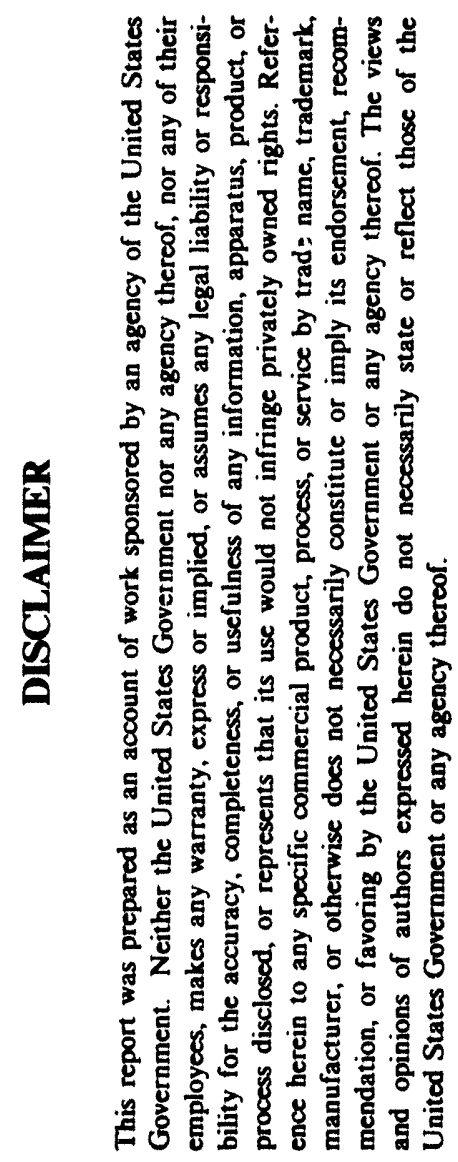

to be published

Proceedings of Hadron 93

Como, Italy

June 21-25, 1993

SEP 291993

OSTI

\section{MASTER}


ORNL-CCIP-93-08

\title{
Hadronic Molecules and Scattering Amplitudes from the Nonrelativistic Quark Model ${ }^{1}$
}

\author{
T.Barnes \\ Physics Division and Center for Computationally Intensive Physics \\ Oak Ridge National Laboratory, Oak Ridge, TN 37831-6373
}

\begin{abstract}
This report summarizes recent calculations of low-energy hadron-hadron scattering amplitudes in the nonrelativistic quark potential model, which assume that the scattering mechanism is a single interaction (usually OGE) followed by constituent interchange. We refer to the scattering diagrams as "quark Born diagrams". For the cases chosen to isolate this mechanism, I=2 $\pi \pi, I=3 / 2 \mathrm{~K} \pi, \mathrm{KN}$ and NN, the results are usually in good agreement with experimental S-wave scattering amplitudes given standard potential-model parameters. These calculations also lead to predictions of vectorvector bound states, one of which may be the $\theta(1710)$. This assignment can be teated by searches for $\mathrm{K} \bar{K} \pi \pi$ and $\phi \pi^{0} \gamma$ decay modes of the $\theta(1710)$.
\end{abstract}

\section{Introduction}

The determination of the strong forces between hadrons in terms of the underlying quark and gluon degrees of freedom has been a long-standing goal of research in QCD. Many theoretical studies at the quark-and-gluon level have considered the important nucleon-nucleon interaction [1], and have been successful in extracting the short-range repulsive core, which they find to be dominated by the OGE spin-spin interaction. Interactions of pseudoscalar mesons have been studied variationally in the nonrelativistic quark potential model [2]; these calculations found qualitatively correct results for S-wave phase shifts, and predicted deuteronlike kaon-antikaon "molecule" bound states, which have been identified with the $f_{0}(975)$ and $a_{0}(980)$ just below $K \bar{K}$ threshold. Although the results of these studies are very encouraging, the nonperturbative techniques used (resonating group methods and many-parameter variational calculations) require considerable theoretical effort to determine hadron scattering amplitudes.

Our collaboration has investigated the possibility that certain low-energy hadron-hadron scattering reactions may be dominated by simple perturbative processes. We consider channels in which s-channel resonances are not expected, and (except for the baryon-baryon case) will discuss reactions involving an external pseudoscalar meson, so one-pion-exchange is forbidden. Through these constraints we hope to isolate and study the mechanism of hadron scattering which does not involve valence annihilation and pair production.

\section{Method}

We assume that nonresonant hadron scattering is dominated by the lowest-order perturbative QCD process, specifically one-gluon-exchange followed by constituent (quark) interchange. Nonperturbative

\footnotetext{
${ }^{1}$ Presented at HADRON93, Como, Italy (21.25 June 1993).
} 
QCD is assumed to contribute to scattering only through the formation of the asymptotic hadron wavefunctions (or in the most detailed study as a single scattering interaction through a linear confining potential [3]). The constituent-interchange scattering mechanism is of course not new [4]. Our contribution to this picture is to couple constituent interchange to OGE in the nonrelativistic quark potential model, and to evaluate the color, spin and spatial matrix elements within this OGE+CI formalism. We use a conventional nonrelativistic quark-model Hamiltonian of the form

$$
H=\sum_{i=1}^{4}\left(\frac{p_{i}^{2}}{2 m_{i}}+m_{i}\right)+\sum_{i<j}\left[V_{\text {conf }}\left(r_{i j}\right)+V_{h y p}\left(r_{i j}\right) \vec{S}_{i} \cdot \vec{S}_{j}\right]\left(\lambda_{i}^{a} / 2\right) \cdot\left(\lambda_{j}^{a} / 2\right),
$$

where $V_{\text {hyp }}=-\left(8 \pi \alpha_{s} / 3 m_{i} m_{j}\right) \delta\left(\vec{r}_{i j}\right)$ is the contact color-hyperfine interaction and $V_{\text {conf }}$ is the spinindependent confining potential. We then calculate the Born-order scattering amplitude and corresponding phase shifts, using relativistic phase space and kinematics for the external hadrons. In most cases discussed here the hyperfine term dominates [3], and we show explicit results for this interaction and use simple Gaussian hadron wavefunctions for illustration unless stated otherwise.

In a two-meson scattering process without identical quarks the OGE + CI scattering mechanism leads to four scattering diagrams, since there are $2 ! \cdot 2 !$ permutations of OGE interactions between the incoming lines. One such diagram is shown below, and the rules for generating and evaluating these diagrams are given in reference [5].

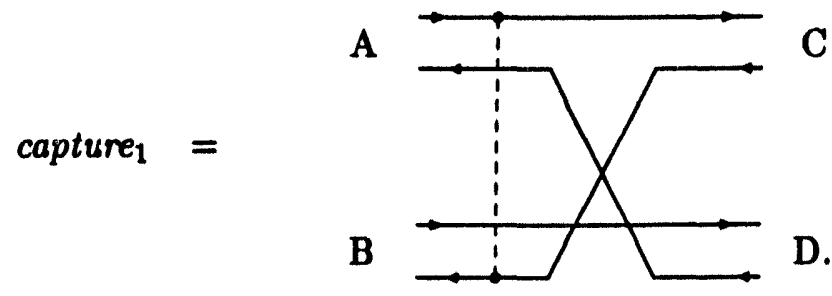

\section{Results for $\pi \pi, \mathbf{K} \pi, \mathbf{V V}, \mathrm{KN}$ and $\mathbf{N N}$}

a) $I=2 \pi \pi$

The $\mathrm{I}=2 \pi \pi$ scattering amplitude due to the contact spin-spin interaction with Gaussian wavefunctions may be evaluated in closed form [5]. The S-wave is dominant at energies studied experimentally, and is given by (2) below. The two free parameters are reasonably well established in quark model phenomenology, $\alpha_{a} / m_{q}^{2} \approx$ $\left(0.6 / 0.33^{2}\right) \mathrm{GeV}^{-2}$ and $\beta_{\pi} \approx 0.3 \mathrm{GeV}$. We fix $\alpha_{s} / m_{q}^{2}$ at this standard value and fit the less well-determined Gaussian width parameter $\beta_{\pi}$ to the data of Hoogland et al. [6]. This is shown in figure 1 , and the fitted value is $\beta_{\pi}=0.337 \mathrm{GeV}$, close to expectations. There is a clear need for an experimental determination of the phase shift above $M_{\pi \pi}=1.5 \mathrm{GeV}$, which we predict to be near an extremum.

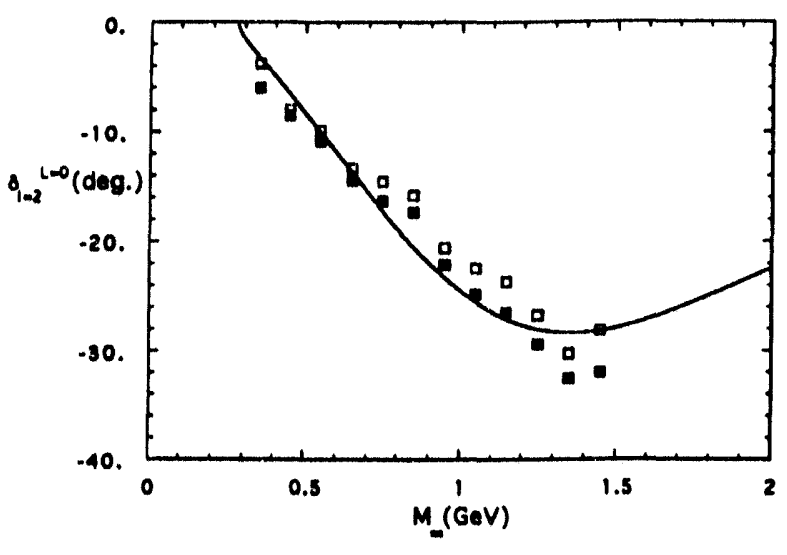

Figure 1: $I=2 \pi \pi \mathrm{S}$-wave phase shift. 
The S-wave phase shift is

$$
\sin \delta_{2}^{(0)}=-\left\{\frac{4 \alpha_{s} \beta_{\pi}^{2}}{9 m_{q}^{2}}\left(1-e^{-\left(s-4 M_{\pi}^{2}\right) / 8 \beta_{\pi}^{2}}+\frac{\left(s-4 M_{\pi}^{2}\right)}{\sqrt{27} \beta_{\pi}^{2}} e^{-\left(s-4 M_{\pi}^{2}\right) / 12 \beta_{\pi}^{2}}\right) / \sqrt{1-4 M_{\pi}^{2} / s}\right\},
$$

which leads to an $I=2 \pi \pi$ scattering length of

$$
a_{2}^{(\pi \pi)}=-\frac{2}{9}\left(1+\frac{8}{\sqrt{27}}\right) \frac{\alpha_{a} M_{\pi}}{m_{q}^{2}} .
$$

This is numerically $a_{2}^{(\pi \pi)} / M_{\pi}^{-1}=-0.059$, in good agreement with Weinstein's (unscaled) variational result [7], and with the PCAC result of Weinberg [8] and the recent parametrization of Morgan and Pennington [9]; the latter two references both find $a_{2}^{(\pi \pi)} / M_{\pi}^{-1}=-0.06$.

b) $I=3 / 2 \mathrm{~K} \pi$

Another annihilation-free channel is $\mathrm{I}=3 / 2 \mathrm{~K} \pi[10]$. Here we have four free parameters, $\alpha_{\Delta} / m_{q}^{2}$, $m_{q} / m_{s}, \beta_{\pi}$ and $\beta_{K}$. We leave $\alpha_{b} / m_{q}^{2}$ and $\beta_{\pi}$ equal to their $\pi \pi$ values. The phase shifts are found to be rather insensitive to the relative kaon/pion length scale, so we set $\beta_{K}=\beta_{\pi}$; this leaves only $m_{q} / m_{\theta}$ undetermined, and typical constituent quark parameters suggest $m_{q} / m_{s} \approx 0.33 \mathrm{GeV} / 0.55 \mathrm{GeV}=0.6$.

When we tuse the S-wave phase shift data of Estabrooks et al.' [1i] to determine $m_{\text {a }}$ we find that the optimum ratio is $m_{q} / m_{s}=0.677$, similar to expectations. Imposing $m_{q} \neq m_{\text {, has }}$ an interesting effect; it induces a $P$-wave amplitude. The theoretical S-wave and P-wave phase shifts are shown in figure 2 . Note that the previous prediction of an extremum in the $I=2 \pi \pi$ S-wave phase shift near $1.5 \mathrm{GeV}$ (in figure 1) also holds for its $S U(3)$-partner $I=3 / 2 K \pi S$ wave phase shift (figure 2). I=3/2 $K \pi$ experimental datis are available at higher invariant mass than $I=2 \pi \pi$, and appear to support this prediction. Our result for the scattering length,

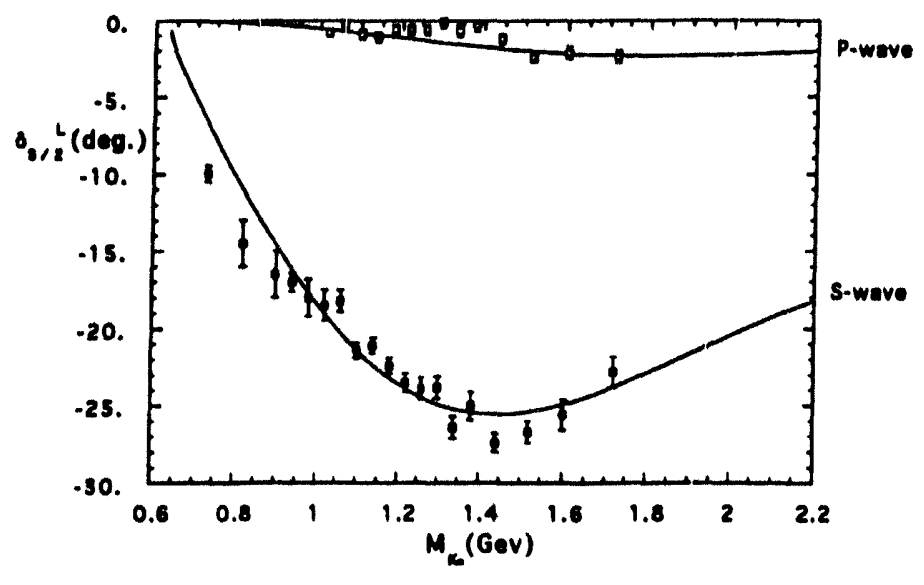

Figure 2: $I=3 / 2 \mathrm{~K} \pi \mathrm{S}$-wave and $\mathrm{P}$-wave phase shifts.

$$
a_{3 / 2}^{(K \pi)}=-\frac{2 \alpha_{s}}{9 m_{q}^{2}\left(M_{\pi}^{-1}+M_{K}^{-1}\right)}\left[1+\left(\frac{4 \beta_{\pi}^{2}}{2 \beta_{\pi}^{2}+\beta_{K}^{2}}\right)^{3 / 2}+\frac{m_{q}}{m_{s}}\left\{\left(\frac{4 \beta_{K}^{2}}{2 \beta_{K}^{2}+\beta_{\pi}^{2}}\right)^{3 / 2}+\left(\frac{2 \beta_{K} \beta_{\pi}}{\beta_{K}^{2}+\beta_{\pi}^{2}}\right)^{3}\right\}\right]
$$

corresponds to about $-0.077 / M_{\pi}$ with our preferred parameter set; this is consistent with the PCAC result of $\approx-0.07 / M_{\pi}[12]$ and with the (rather wide) range of reported experimental values. Finally, the predicted and observed $P$-waves are qualitatively consistent.

\section{c) Other meson-meson channels; vector-vector molecules}

I=1 KK scattering may be treated using essentially identical techniques [5]. We find a moderately strong repulsive interaction, which agrees with the variational results of Weinstein [7]. Unfortunately there are no experimental results for this reaction.

Although these analytical techniques have not yet been applied in detail to other meson-meson channels, Swanson [3] has carried out similar numerical studies of the phenomenologically interesting 
vector-vector and vector-pseudoscalar channels with $u, d$ and $s$ quarks. The interesting question in these channels is whether unusual states such as the $\theta(171.0)$ and the $f_{1}(1420)$ might actually be weakly-bound molecular vector-vector bound states or $K^{*} K$ threshold enhancements, respectively.

Bound states form most easily in strongly-coupled systems that are nearly degenerate, since there are small energy denominators, and one linear combination of states in a $2 \times 2$ Hamiltonian with an off-diagonal interaction always experiences an attractive interaction. The $q s \bar{q} \bar{s}$ vector-vector system is such a case; here Swanson finds an especially large $K^{*} \overline{\mathbf{K}}^{*} \leftrightarrow \omega \phi$ matrix element, which in a multichannel Schrödinger formalism described by Dooley, Swanson and Barnes [13] leads to scalar and tensor bound states. These vector-vector molecules might be identified with the $\theta(1710)$; similar suggestions for this and other unusual resonances have appeared in the literature [14,15, 16]. The couplings and branch.ıg fractions of the linear combination $|\theta\rangle=\left(\left|\mathrm{K}^{*} \overline{\mathrm{K}}^{*}\right\rangle+|\omega \phi\rangle\right) / \sqrt{2}$ which is formed by a large off-diagonal interaction explain many of the unusual properties of the $\theta$. New predictions of this model [13] include a large branching fraction to $\mathrm{K} \overline{\mathrm{K}} \pi \pi(B . F . \approx 35 \%)$, an unusual $\phi \pi^{0} \gamma$ mode $(B . F . \approx 0.3 \%)$, $B . F . K \bar{K} / B . F . \eta \eta \approx 2$, a weak $\pi \pi$ mode $($ S.F. $\sim 5-10 \%$ ), the $\psi$-hadronic ratio $(\psi \rightarrow \phi \theta) /(\psi \rightarrow \omega \theta) \approx$ $2 / \lambda^{2}$ (just as for a $K \bar{K}$ molecule), and a small $\gamma \gamma$ width [17].

d) $\mathrm{KN}$

One may also study meson-baryon and baryon-baryon scattering using quark Born diagrams. We have considered KN scattering in detail [18], since there are no valence annihilation contributions and the low partial waves have been studied experimentally. In $\mathrm{KN}$ scattering there are $\mathrm{I}=0$ and $\mathrm{I}=1$ channels, and both are repulsive, with $\mathrm{I}=1$ having the strongest interaction.

These features of KN interactions are correctly predicted by the quark Born formalism. The welldetermined $I=1 \mathrm{KN}$ scattering length of $\approx-0.33 \mathrm{fm}$ is predicted to be about $\mathbf{- 0 . 3 5}$ fm given standard quark model parameters (dashed). The $\mathrm{I}=0$ scattering length is predicted to be about $-0.12 \mathrm{fm}$, but is unfortunately not yet well determined experimentally. At higher energies we find that the Born-order S-wave phase shifts are too "soft"; the predicted phase shifts are largest at $P_{c m} \approx 0.5 \mathrm{GeV}$, whereas the observed ones appear to grow monotonically to the maximum experimental momentum of $P_{c m} \approx 0.7 \mathrm{GeV}$. This may be an artifact of our "soft" Gaussian baryon wavefunctions, since a good fit is possible given a moderately reduced nucleon length scale (solid). Higher $\mathrm{KN}$ partial waves are also interesting in that they show clear evidence of spin-

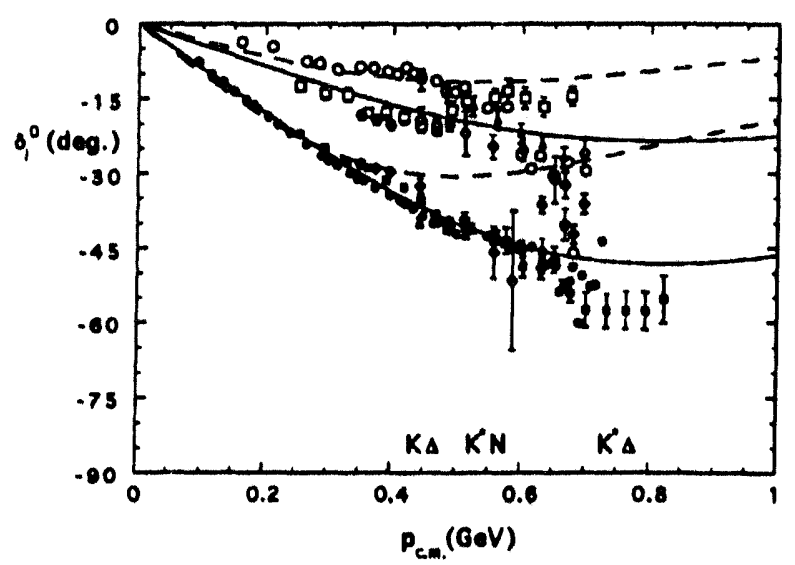

Figure 3: KN S-wave phase shifts; $I=0$ (open points) and $I=1$ (solid points). orbit interactions, which we have not yet incorporated in our formalism.

e) NN

Finally, the short-range repulsive core in the NN interaction provides an important test of any description of hadron-hadron scattering. We assume that the repulsive core is dominantly due to the OGE spin-spin hyperfine interaction, which was a conclusion of resonating group and variational studies [1]. We do not expect to reproduce the longer-ranged attraction, which is variously attributed to spatial wavefunction distortion or meson exchange; neither effect is present in our formalism at Born order.

When modelled as a potential the short-distance core is typically found to have a maximum of $\approx 1$ $\mathrm{GeV}$ and a range of about $1 / 2 \mathrm{fm}$ for both $\mathrm{NN}$ isospin states. In our formalism this is a straightforward 
calculation [19], and the two parameters $\alpha_{a} / m_{q}^{2}$ and $\alpha_{N}$ (baryon Gaussian width parameter) are already determined. The low-energy NN potentials we find are shown in figure 4 .

These core potentials are very similar to the re-

sults of resonating group calculations. Thus, our rather surprising conclusion is that the cores are dominantly Born-order one-gluon-exchange effects. The Born-order S-wave NN phase shifts in contrast are quite inaccurate, since the potentials are so large. We have used numerical integration of the Schrödinger equation to determine phase shifts which result from the Born-order NN core potentials, and the resulting phase shifts closely resemble those of Oka and Yazaki [1].

In a search for possible molecule states we investigated other nonstrange baryon-baryon channels. We found that the $I=0, S=1 \Delta \Delta$ channel has an attractive core and might form a bound state. However, the assumptions of hyperfine dominance and the single-

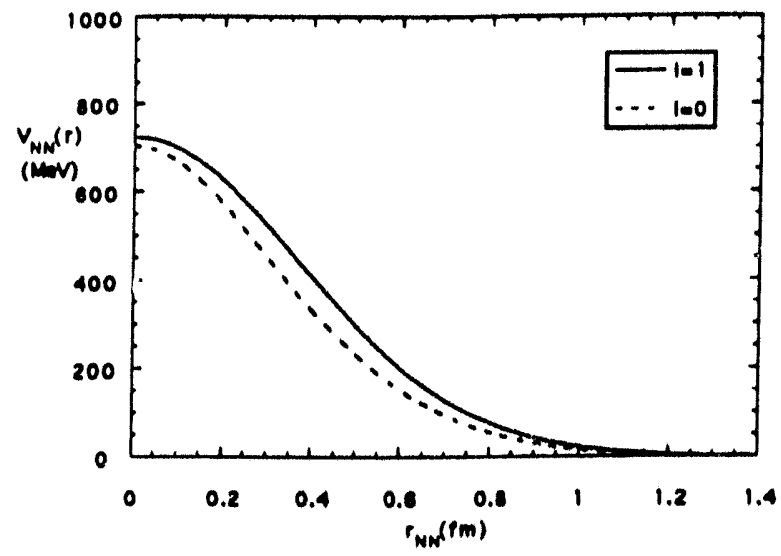

Figure 4: $I=0$ and $I=1$ low-energy NN potentials. channel approximation have not been tested for this system and may modify our conclusion about bound states, since a fall-apart coupling from $I=0, S=1$ $\Delta \Delta$ to $N N$ is present.

\section{Surnmary and Conclusions}

We have found that a simple perturbative mechanism, one-gluon-exchange followed by constituent interchange, leads to an accurate description of low energy hadron-hadron scattering in several annihilationfree channels, given standard quark-model parameters. In future work it will be interesting to generalize these techniques through 1) the inclusion of other terms such as spin-orbit (needed for KN higher partial waves), 2) the use of more realistic baryon wavefunctions (for $\mathrm{KN}$ at higher energies), and 3 ) the incorporation of $q \bar{q}$ annihilation, which is known to be a very important effect when allowed, as in $\mathrm{K} \bar{K}$ and $I=0 \pi \pi$ scattering. With these improvements we expect that it will be possible to predict phase shifts and bound states accurately in other meson-meson and meson-baryon channels, which will be accessible to experiments at hadron facilities such as CEBAF, DAPHNE, KAON, KEK and LEAl. Our comparisons with experiment suggest that better determinations of some scattering amplitudes, such as $\mathrm{I}=2 \pi \pi$ (above $1.5 \mathrm{GeV}$ ), I=3/2 $\mathrm{K} \pi$ and especially $\mathrm{I}=0 \mathrm{KN}$, would be very useful contributions to the study of soft hadron scattering.

\section{Acknowledgements}

It is a pleasure to acknowledge the efforts of the organisers of this meeting, in particular the co-chairmen T.Bressani and G.Preparata. The advice and essential contributions of my collaborators S.Capstick, K.Dooley, G.Grondin, N.Isgur, M.D.Kovarik, E.S.Swanson and J.Weinstein are also gratefully acknowledged. This research was sponsored in part by the United States Department of Energy under contract DE-AC05-840R21400, managed by Martin Marietta Energy Systems, Inc. 


\section{References}

[1] D.A.Liberman, Phys. Rev. D16, 1542 (1977); C.E.DeTar, Phys. Rev. D17, 302 (1977); ibid., p.323; M.Oka and K.Yazaki, Phys. Lett. 90B, 41 (1980); Prog. Theor. Phys. 66, 556 (1981); ibid., p.572; A.Faessler, F.Fernandez, G.Lubeck and K.Shimizu, Phys. Lett. 112B, 201 (1982); K.Maltman and N.Isgur, Phys. Rev. Lett. 50, 1827 (1983); M.Oka and C.J.Horowitz, Phys. Rev. D31, 2773 (1985); Y.Koike, Nucl. Phys. A454, 509 (1986); K.Shimizu, Rep. Prog. Phys. 52, 1 (1989).

[2] J.Weinstein and N.Isgur, Phys. Rev. Lett. 48, 659 (1982); Phys. Rev. D27, 588 (1983); Phys. Rev. D41, 2236 (1990); Phys. Rev. D43, 95 (1991).

[3] E.S.Swanson, Ann. Phys. (NY) 220, 73 (1992).

[4] See for example G.P.Lepage and S.J.Brodsky, Phys. Rev. D22, 2157 (1080), and the review by S.J.Brodsky and G.P.Lepage in Perturbative Quantum Chromodynamics, ed. A.Muller (World Scientific, 1989).

[5] T.Barnes and E.S.Swanson, Phys. Rev. D46, 131 (1992); for closely related work on mesonmeson scattering see B.Masud, J.Paton, A.M.Green and G.Q.Liu, Nucl. Phys. A528, 477 (1991); D.Blaschke and G.Röpke, Phys. Lett. B299, 332 (1993) .

[6] W.Hoogland et al., Nucl. Phys. B126, 109 (1977).

[7] J.Weinstein, Phys. Rev. D47, 911 (1993).

[8] S.Weinberg, Phys. Rev. Lett. 17, 616 (1966).

[9] M.R.Pennington, personal communication; see also K.L.Au, D.Morgan and M.R.Pennington, Phys. Rev. D35, 1633 (1987); D.Morgan and M.R.Pennington, Phys. Lett. 258B, 444 (1991).

[10] T.Barnes, E.S.Swanson and J.Weinstein, Phys. Rev. D46, 4868 (1992).

[11] P.Estabrooks et al., Nucl. Phys. B133, 490 (1978); at lower energies see also B.Jongejans et al., Nucl. Phys. B67, 381 (1973); C.B.Lang and W.Porod, Phys. Rev. D21, 1295 (1980).

[12] S.Weinberg, Phys. Rev. Lett. 17, 616 (1966); Y.Tomozawa, Nuovo Cim. 46A, 707 (1966); J.A.Cronin, Phys. Rev. 161, 1483 (1967); R.W.Griffith, Phys. Rev. 176, 1705 (1968).

[13] K.Dooley, E.S.Swanson, and T.Barnes, Phys. Lett. 275B, 478 (1992); K.Dooley, in Proceedings of the 4th International Conference on Hadron Spectroscopy "Hadron '91" (College Park, Md. 12-16 August 1991), (World Scientific, 1992), pp.789-794; K.Dooley, Ph.D. thesis, University of Toronto (1993).

[14] Yu.S.Kalashnikova, in Proceedings of the 4th International Conference on Hadron Spectroscopy "Hadron '91" (College Park, Md. 12-16 August 1991), (World Scientific, 1992), pp.777-782.

[15] N.Törnqvist, ibid., pp.795-798; Phys. Rev. Lett. 67, 556 (1991); see also contribution in these procetdings.

[16] T.E.O.Ericson and G.Karl, CERN report CERN-TH-6808/93 (March 1993); G.Karl, in Proceedings of the Second Biennial Conference on Low Energy Antiproton Physics (Courmayeur, 1992).

[17] E.S.Ackleh, K.Dooley and Z.P.Li, University of Toronto report UTPT-92-03. 
[18] T.Barnes and E.S.Swanson, MIT / ORNL report MIT-CTP-2169 / ORNL-CCIP-92-15.

[19] T.Barnes, S.Capstick, M.D.Kovarik and E.S.Swanson, CEBAF / MIT / ORNL rejort CEBAF93-04 / MIT-CTP-2187 / ORNL-CCIP-93-01, to appear in Phys. Rev. C (1 August 1993). 

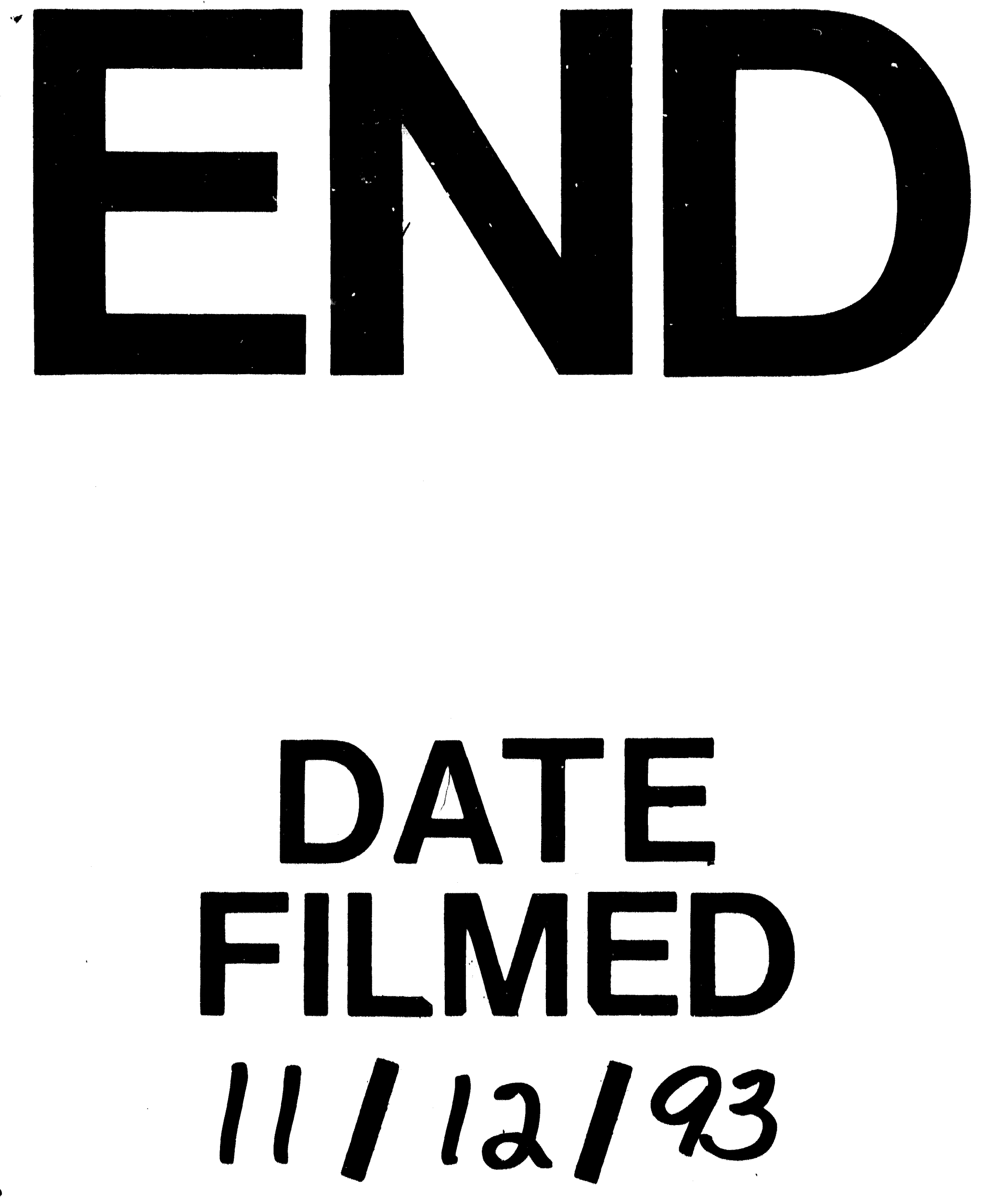
